IRA-International Journal of Applied Sciences ISSN 2455-4499; Vol.05, Issue 03 (2016)

Institute of Research Advances

Pg. no. 129-146

http://research-advances.org/index.php/IRAJAS

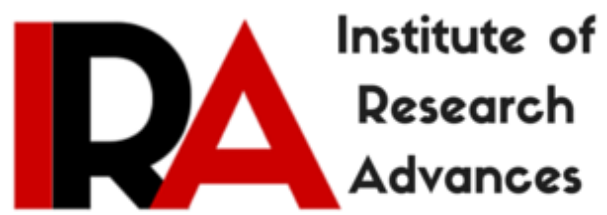

\title{
CW Optical Limiting Study in Disperse Yellow Dye-doped PMMA-MA Polymer Films
}

\author{
Shubrajyotsna Aithal ${ }^{1}$, P. S. Aithal ${ }^{2}$ and G. K. Bhat ${ }^{3}$ \\ ${ }^{1}$ Research Scholar, Dept. of Chemistry, Rayalaseema University, \\ Kurnool -518007, Andhra Pradesh, India. \\ ${ }^{2}$ Srinivas Institute of Management Studies, \\ Pandeshwar, Mangalore - 575001, India. \\ ${ }^{1,3}$ Dept. of Chemistry, Srinivas Institute of Technology, \\ Valachil, Mangalore, 574143, India.
}

Type of Review: Peer Reviewed.

DOI: http://dx.doi.org/10.21013/jas.v5.n3.p4

\section{How to cite this paper:}

Aithal, S., Aithal, P., \& Bhat, G. (2016). CW Optical Limiting Study in Disperse Yellow Dye-doped PMMA-MA Polymer Films. IRA-International Journal of Applied Sciences (ISSN 2455-4499), 5(3), 129-146. doi:http://dx.doi.org/10.21013/jas.v5.n3.p4

(C) Institute of Research Advances

\section{(c) EY-NO}

This work is licensed under a Creative Commons Attribution-Non Commercial 4.0 International License subject to proper citation to the publication source of the work.

Disclaimer: The scholarly papers as reviewed and published by the Institute of Research Advances (IRA) are the views and opinions of their respective authors and are not the views or opinions of the IRA. The IRA disclaims of any harm or loss caused due to the published content to any party. 


\section{ABSTRACT}

Molecules with high two-photon absorption (TPA) cross section and the molecules which have reverse saturation absorption (RSA) cross section are of great interest today because of their application in three-dimensional optical data storage and photonic switches. During last forty years, several techniques have been developed to determine the two-photon absorption crosssections and reverse saturation absorption cross-sections of the materials. This includes direct methods, such as nonlinear transmission and Z-scan methods and indirect methods, such as twophoton excited fluorescence and two-photon pump-probe transient absorption spectroscopy. During recent years, the organic dye-doped polymer films getting more attention due to their advantages to fabricate photonics devices. In this paper, we have studied the nonlinear optical properties like nonlinear absorption and nonlinear refraction of an azo dye Disperse yellow-7 (DY-7) doped in Polymethyl methacrylate-methacrylic acid (PMMA-MA) polymer matrix using open aperture and closed aperture Z-scan experimental methods using continuous wave (CW) laser. The optical limiting properties of these films are also studied in Type 1 and Type 2 configurations at different input power using continuous wave (CW) laser beams of $532 \mathrm{~nm}$ wavelength.

Keywords: Organic Nonlinear material, Dye-doped polymer films, Optical limiting, Open and closed aperture $\mathrm{Z}$ scan.

\section{Introduction}

The search for optimum nonlinear material is active since forty years, through the combined efforts of physics, material science, and chemistry researchers by carrying out continuous nonlinear experiments on various possible materials and this field is very active in research and a promising tool for future photonics technology. Many organic dyes have shown considerable third order nonlinearity for intense laser light like Phthalocynamines (Perry et, al, 1994, [1]), Porphyrins (Blau et, al, 1985 [2], Sevian et. al. 1996 [3]), Rhodamine 6G (Sharma et. al. 2008, [4]. Castillo et al (1994 [5]) observed the thermal lensing effect resulting from one and twophoton absorption. Nonlinear characterization of Mercurochrome dye is studied for potential application in optical limiting by Krishnamurthy et. al. 2010 [6]. Rekha et. al. 2009 studied nonlinear characteristic and optical limiting effect of oil red $\mathrm{O}$ azo dye in liquid and solid media [7]. Muto et al (1998 [8]) shown that the nonlinear response of disperse red1 (DR1) dye with PMMA at $532 \mathrm{~nm}$ increases with increasing concentration up to $1 \mathrm{wt} \%$ of DR1 in PMMA and decreases for larger concentration. Zidan, et al (2011) [9] have studied the optical limiting behaviour of disperse red 1 dye-doped polymer. Brzozowski et al 2001 [10]) reported the nonlinear response of pseudo-stilbene type azobenzene dye embedded in a polymer (PMMA, poly (1,4-phenylene vinylene)) matrix by Z- scan technique. Third order optical nonlinearity and optical limiting studies of propane hydrazides is reported by Naseema, K et. al. 2012 [11).

Venugopal Rao et al (2002) [12] reported the non-linearity of Rhodamine B dye in methanol. Umakanta Tripathy et al (2002) [13] published a few parameters of IR 140 dye in dimethyl sulphoxide (DMSO) using Z-scan techniques. Third order optical nonlinearities and spectral characteristics of a dye methylene blue are studied by Sukumaran et al (2011) [14]. Chen et al (2003) [15] reported the third-order optical nonlinearity of fullerene-containing polyurethane films at telecommunication wavelengths. Zidan et al (2011) investigated the optical limiting properties of acid blue-29 in various solvents [16]. Dharmadhikari et al (2004) observed the higher-order optical nonlinearities in 4-dimethylamino-N-methyl-4-stilbazoliumtosylate [17] and Ganeev et al (2004) reported the fifth order optical nonlinearity of pseudoisocyanine solution [18]. Del Nero et al (2005) determined the non-linear refractive index of methyl orange in acetone 
under different PH condition by using Z- scan technique [19]. Hassan et al 2015 [20] studied optical limiting properties of sudan red B in solution and solid film. R. P. Singh et. al. 2003 studied two photon absorption cross section and nonlinear absorption properties of DASPB [21], DO-25, and DY-7 [22] at picoseconds regime in solution form. Many other studies have been reported on use of dye-doped polymer films for optical phase conjugation through degenerate four waves mixing (DFWM) using low power CW lasers [23-30]. The optical third harmonic nonlinearity in organic materials generally originated by two photon and multiphoton absorption, two photon florescence, excited state absorption, reverse saturation absorption, thermal lensing effect etc. Reports show that there have been less work carried on the study of nonlinearities of dyes in solid medium. It is expected that by studying nonlinear properties of dyes in solid medium and identifying active and efficient nonlinear dyes for low power $\mathrm{CW}$ laser beams, one can fabricate new elements/components, which have potential applications in optical limiting and optical switching.

\section{Ideal Optical Limiter}

An ideal limiter is a device which shows linear transmission characteristics below a threshold level and fixes the output to a constant level above it, thus providing safety protection to sensors or human eyes. An ideal optical limiter is a photonic device or component which has ideal optical limiting characteristics. It can take any intensity input laser beam both continuous wave $(\mathrm{CW})$ or pulsed wave of any time duration. It has to process such incident light beam internally using nonlinear properties of the medium and provide output laser beam of constant intensity or fluency. The ideal optical limiter has the characteristics shown in Fig. 1. It has a high linear transmission for low input (e.g. energy $\mathrm{E}$ or power $\mathrm{P}$ ), a variable limiting input $\mathrm{E}$ or $\mathrm{P}$, and a large dynamic range defined as the ratio of the $\mathrm{E}$ or $\mathrm{P}$ at which the device damages (irreversibly) to the limiting input. Such devices can also be used as power or energy regulators. However, since the primary application of the optical limiter is for sensor protection, and damage to detectors is almost always determined by fluence or irradiance, these are usually the quantities of interest for the output of the limiter. Getting the response of ideal limiter at least above certain minimum input energy turns out to be possible using a wide variety of materials, however, it is very difficult to get the limiting threshold as low as is often required and at the same time have a large dynamic range. Because high transmission for low inputs is desired, the limiter material must have low linear absorption. Some of the characteristics predicted for ideal limiter are:

(1) An ideal limiter device should capable of taking input light beam of any intensity without any material damage.

(2) An ideal limiter device should capable of accepting input light beam without any reflection or scattering from incident surface.

(3) Any variation in the input intensity or power between zero to infinity should maintain constant output intensity irrespective of input intensity variations.

(4) An ideal optical limiter shows linear transmission characteristics below a threshold level and fixes the output to a constant level above it.

(5) The transmission characteristics of an ideal limiter vary depending upon the incident intensity due to nonlinear properties of the limiter material.

(6) The output intensity/fluency of ideal optical limiter is independent on the wavelength and pulse duration of the laser beam.

(7) An ideal optical limiter should limit the input light of wavelength throughout the electromagnetic spectrum. i.e., it should have infinite bandwidth.

(8) The nonlinear material medium used for fabrication of ideal limiter should have very high nonlinearity for entire bandwidth. 
(9) The nonlinear material medium used for fabrication of ideal limiter should limit any laser beam of any power of both CW and pulsed.

(10) An ideal limiter should provide constant output in any environmental conditions like changes in temperature, pressure, and aging.

(11) The construction of ideal limiter should be easy and of low cost.

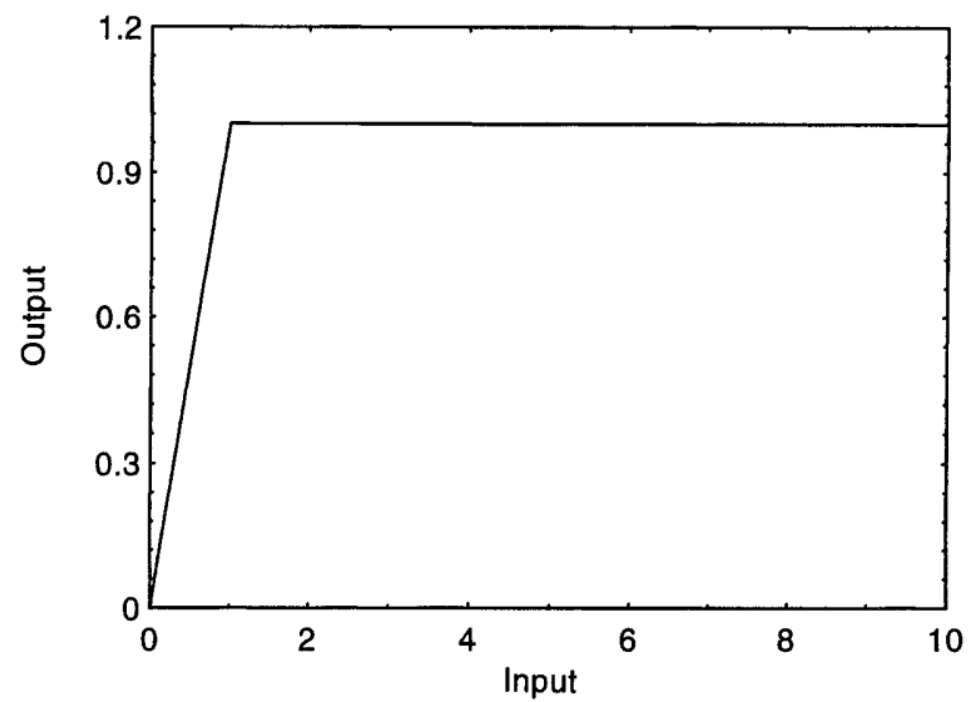

Fig. 1 : The ideal optical limiter input-output response characteristics [31].

The ultimate objective of any optical nonlinear materials research is to find a suitable material which can show optical limiter characteristics close to ideal optical limiter characteristics.

\section{Fabrication \& Linear Optical Properties of DY-7 doped Polymer films}

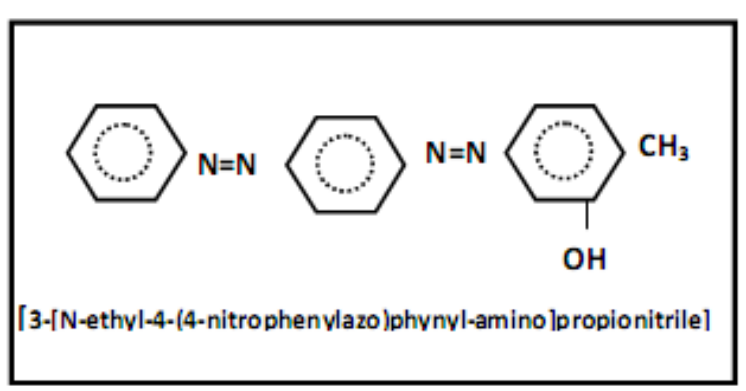

Fig. 2 : (a) Molecular structure of DY-7.

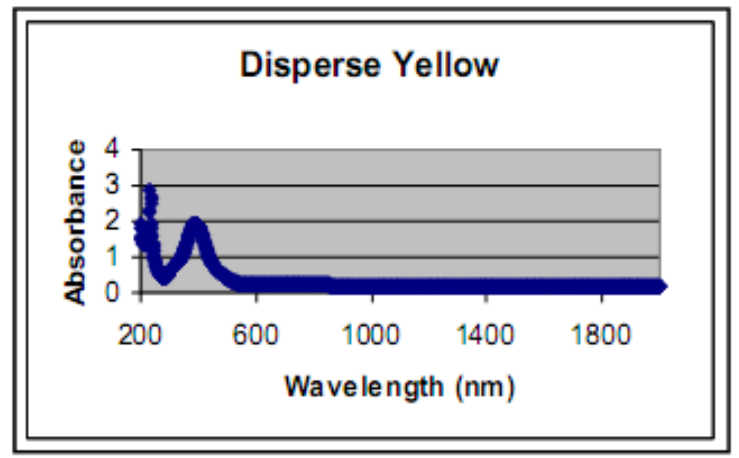

(b) Linear absorption spectrum of DY-7.

Commercially available Disperse Yellow-7 (DY-7) (Aldrich Chemical Co.) is purified by recrystallization twice with spectrograde ethanol and by vacuum sublimation. The purity is determined spectroscopically. Purified chloroform is used as the solvent. To prepare the film, Polymethyl methacrylate - metacrylic acid is used as a polymer matrix. The thin films of DY-7 doped in PMMA-MA are prepared using hot press technique [32]. Thin films of thickness (10 $\mu \mathrm{m}$ ) with $1 \mathrm{mM}, 2 \mathrm{mM}$, and $5 \mathrm{mM}$ dye concentrations are prepared between two glass slides and are used as samples for optical nonlinearity study and optical limiting experiments. 
The linear absorption spectrum of DY-7 doped in PMMA-MA is measured on a VARIAN Cary UV-vis-IR recording Spectrophotometer. The figure 2(b) shows the linear absorption spectrum of the film. The spectral curve has shown that there is a strong absorption band with peak absorption located at $468 \mathrm{~nm}$ with a bandwidth of $80 \mathrm{~nm}$, a medium absorption peaked at $270 \mathrm{~nm}$ with a bandwidth of $60 \mathrm{~nm}$ and no linear absorption is observed in the entire spectral range of 580 to $1200 \mathrm{~nm}$ [33-34]. The Z-scan technique is used to study the nonlinear optical properties of the sample [35-36]. The linear absorption coefficient $\alpha_{0}$ is determined for the chosen wavelength 532 nm by using formula $\alpha_{0}=-\frac{1}{t} \ln \left[\frac{1}{T}\right]$

where $(t)$ is the thickness of sample and $\mathrm{T}$ is the transmittance.

The refractive index $\mathrm{n}_{0}$ can be found from transmittance spectrum of the film according to the following equation

$n_{0}=\frac{1}{\mathrm{~T}}+\left[\left(\frac{1}{\mathrm{~T}^{2}}-1\right)\right]^{1 / 2}$

The linear absorption coefficient and refractive index are shown in table 1.

\section{Nonlinear Absorption using Open Aperture Z-scan :}

The block diagram of the experimental setup used for the open aperture Z-scan study of DY-7 doped in PMMA-MA film is shown in figure 3. A CW diode laser of wavelength 532nm is used as the light source. The Gaussian profiled laser beam is focused by a lens (L1) of focal length $\mathrm{f}=$ $3.5 \mathrm{~cm}$ to produce a beam waist $\omega_{0}$ of $15 \mu \mathrm{m}$. The Rayleigh condition, diffraction length $\mathrm{z}_{\mathrm{R}}=$ $\pi \omega_{0}{ }^{2} / \lambda>\mathrm{L}$ is satisfied in this case so that the sample can be considered as a thin medium, where $\mathrm{L}$ is the thickness of the sample and $\lambda$ is the free space wavelength of the laser beam. The diffraction length for the experimental setup is found to be $2.5 \mathrm{~mm}$. The input power adjusted and noted by means of a convex lens (L3) and Photo detector (P.D.1). The DY-7 dye-doped polymer sample is translated across the focal region of lens L1 along the axial direction that is the direction of the propagation of the laser beam.

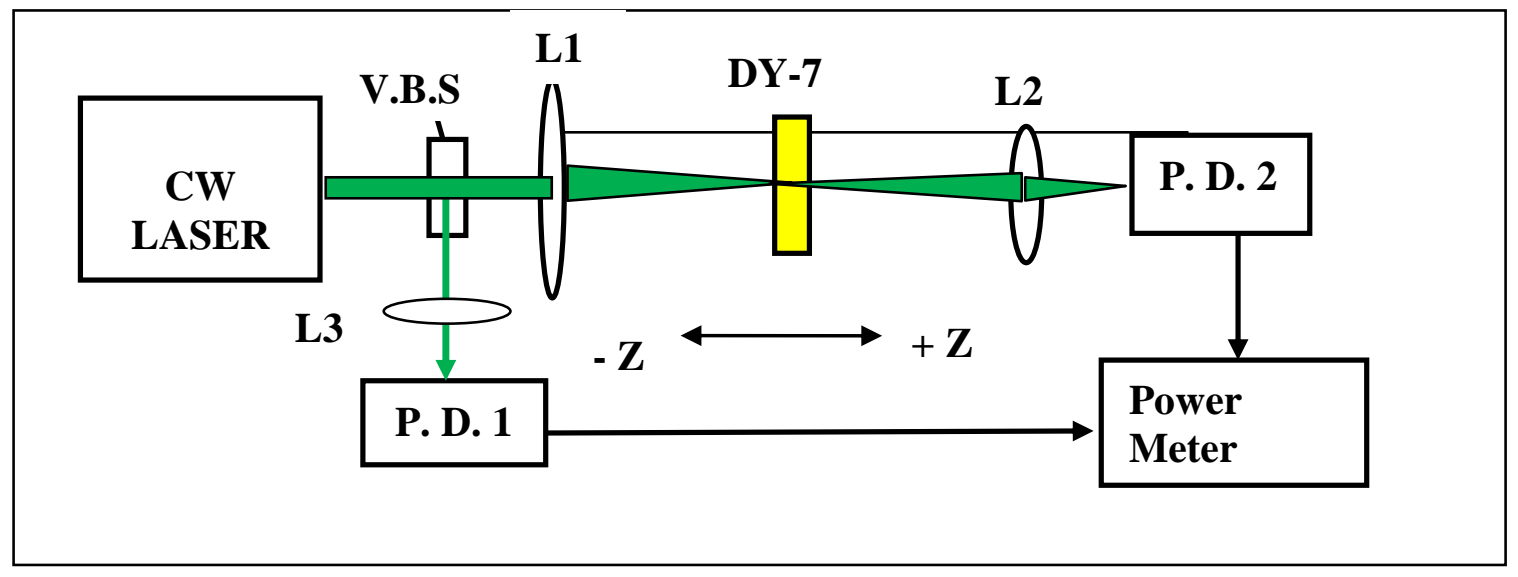

Fig. 3 : Experimental setup for open aperture Z-scan for DY-7 samples.

The transmitted beam is collected by means of a convex lens (L2) and the output intensity is measured using photo detector (P.D.2) fed to the digital power meter. In open aperture $(\mathrm{S}=1)$ configuration, the system is insensitive to nonlinear refraction and can be used to measure the nonlinear absorption cross section. Such Z-scan trace with no aperture is expected to be 
symmetric with respect to the focus $(Z=0)$, where the minimum transmittance (e.g., multiphoton absorption) or a maximum transmittance (e.g., saturation of absorption) occurs. The nonlinear coefficient can be easily calculated from Z-scan transmittance curve.

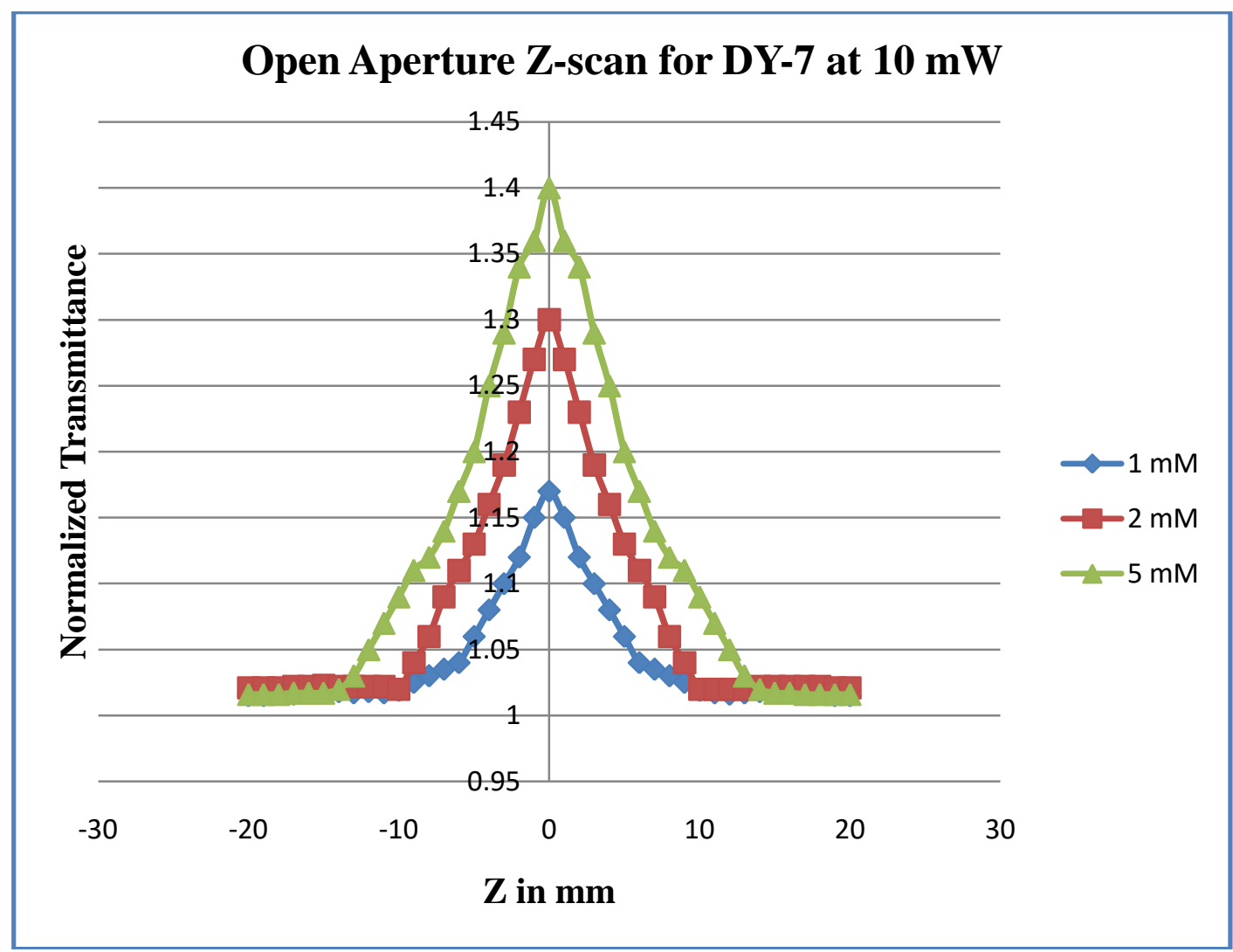

Fig. 4 : CW Open aperture Z-scan plot of DY-7 at different dye concentrations using $532 \mathrm{~nm}, 10$ $\mathrm{mW}$ laser beam.

The Z- scan experiment is performed for DY-7 dye-doped PMMA-MA polymer films of the dye concentration $1 \mathrm{mM}, 2 \mathrm{mM}$, and $5 \mathrm{mM}$ using $532 \mathrm{~nm}$ laser beam at $10 \mathrm{~mW}, 20 \mathrm{~mW}$ and $30 \mathrm{~mW}$ input power. The results are depicted in Fig. 4, to Fig. 6 respectively. In open aperture Z-scan, DY-7 has shown a decrease in transmittance with an increase in irradiance/input intensity due to reverse saturation absorption [37-39].

It is seen from the Z-scan plot that the DY-7 shows strong saturable absorption at low input intensity of laser beam. Even if the linear transmittance of the sample is low (transmittance $\mathrm{T}=$ 0.58), it is observed that due to nonlinear absorption, the transmittance of the sample is increased initially with the increase in intensity. But it is interesting to note that, the saturation absorption (SA) is overtaken by reverse saturation absorption (RSA) effect at higher input intensity (figure 6). The transformation from SA to RSA with the increase in intensity level of the laser is observed in some organic dyes including DY-7 and this behavior can be used for optical limiting as well as optical switching.

Based on open aperture Z-scan plots of DY-7 for different concentrations and at different input power, it is observed that: 
(i) At low input power, saturation absorption (SA) increased with increase in the concentration of dye in the sample.

(ii) At the higher intensity of input light, DY-7 has shown reverse saturation absorption (RSA) so that saturation absorption (SA) of the sample is decreased.

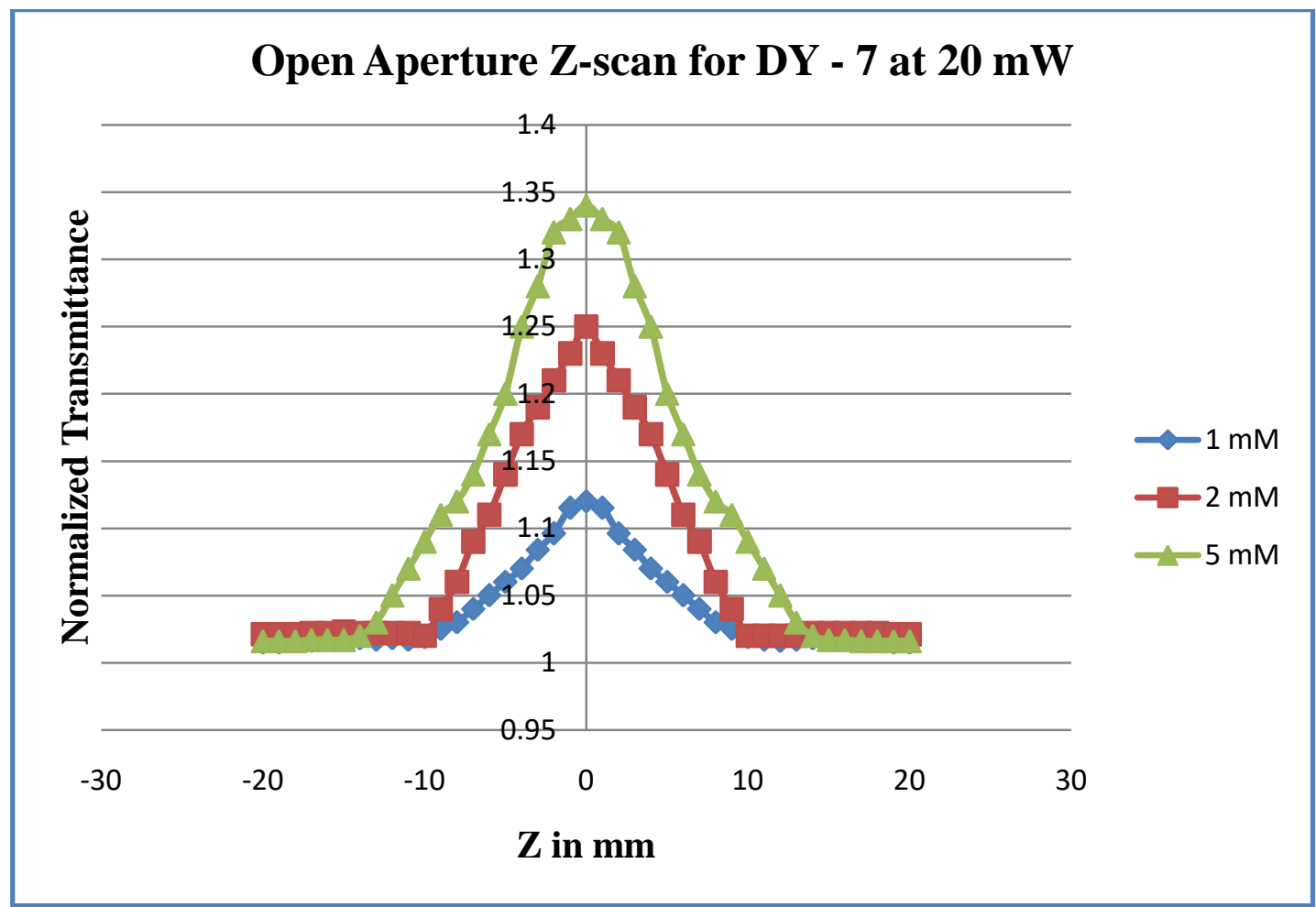

Fig. 5 : CW Open aperture Z-scan plot of DY-7 at different dye concentrations using $532 \mathrm{~nm}, 20$ $\mathrm{mW}$ laser beam.

Reverse saturable absorption is observed in the open aperture Z-scan trace for DY-7 dye in PMMA-MA matrix as it shows minimum transmittance. The nonlinear absorption coefficient $\beta$ can be estimated from the open aperture Z-scan data, where $\beta=(2 \sqrt{2} \Delta T) /\left(I_{0} L_{\text {eff }}\right)$----- (3)

$\mathrm{I}_{0}$ is the intensity at the focal spot given by $I_{0}=2 P_{\text {peak }} / \pi \omega_{0}^{2}$

The effective length of the sample, can be determined from the formula $L_{e f f}=\left(1-e^{-\alpha_{0} L}\right) / \alpha_{0}$ ------ (5)

The transmittance increases with the increasing excitation intensity and has a maximum value at the focus, which is the signature of saturation absorption according to Sheik-Bahae's theory. When saturation absorption occurs, the absorption coefficient $\beta$ is no longer a constant. Instead, it becomes a function of the excitation intensity as in the relation,

$\alpha=\alpha_{0}+\mathrm{I} \beta$------ (6),

Where $\alpha_{0}$ is the linear absorption coefficient, $\alpha$ is the total absorption coefficient, $\beta$ is the nonlinear absorption coefficient, and I is the incident intensity of the laser beam.

In the simplest case, when only third-order nonlinearities are considered in the sample, the resultant change in refractive index becomes: $n=n_{0}+n_{2} I$.

Where $\mathrm{n}$ is the total refractive index of the sample, $\mathrm{n}_{0}$ is the linear refractive index of the sample, $\mathrm{n}_{2}$ is the third order nonlinear refractive index of the sample, and I is the excitation intensity. 


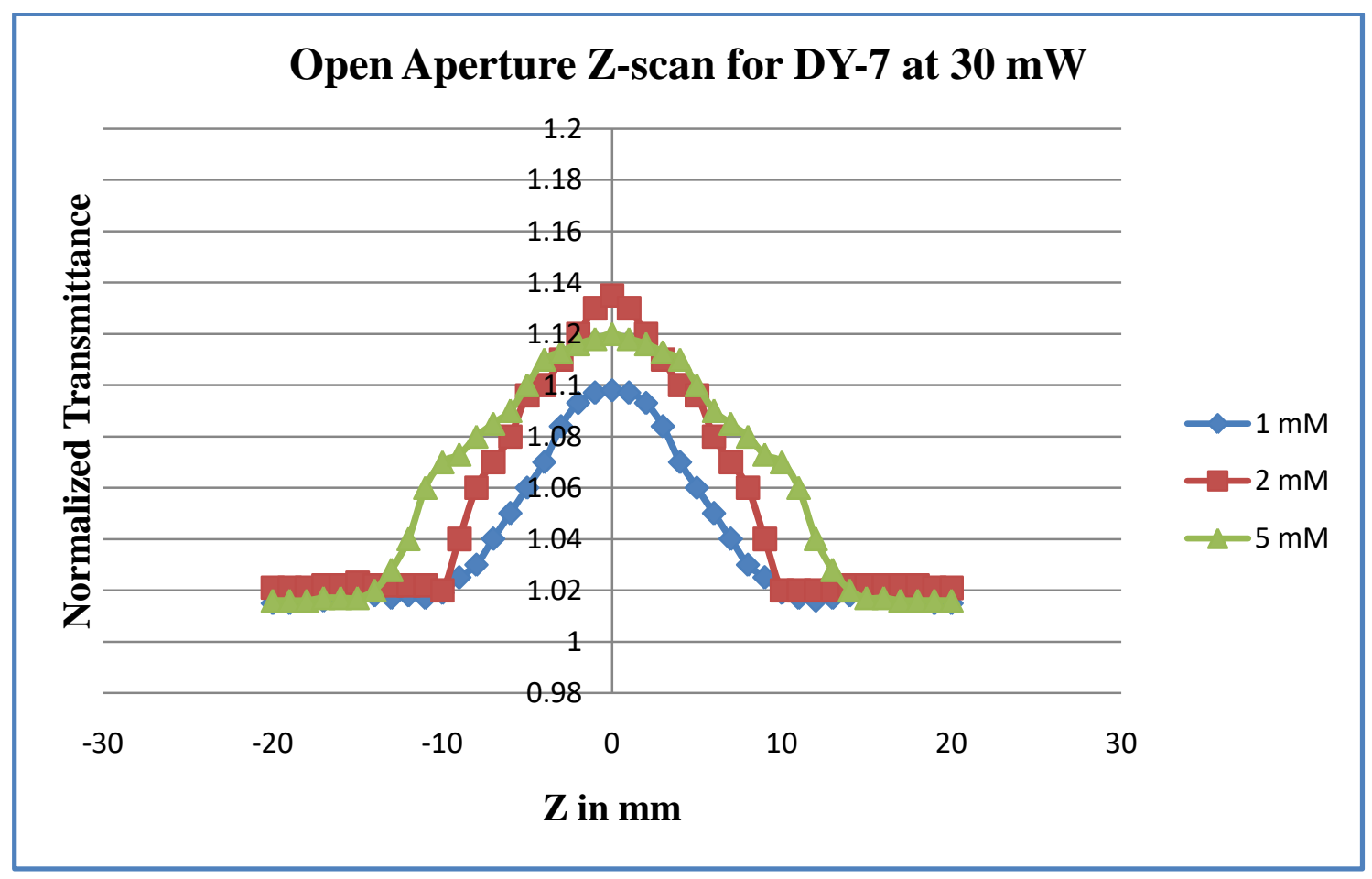

Fig. 6 : CW Open aperture Z-scan plot of DY-7 at different dye concentrations using $532 \mathrm{~nm}, 30$ $\mathrm{mW}$ laser beam.

\section{Nonlinear Refraction using Closed Aperture Z-scan}

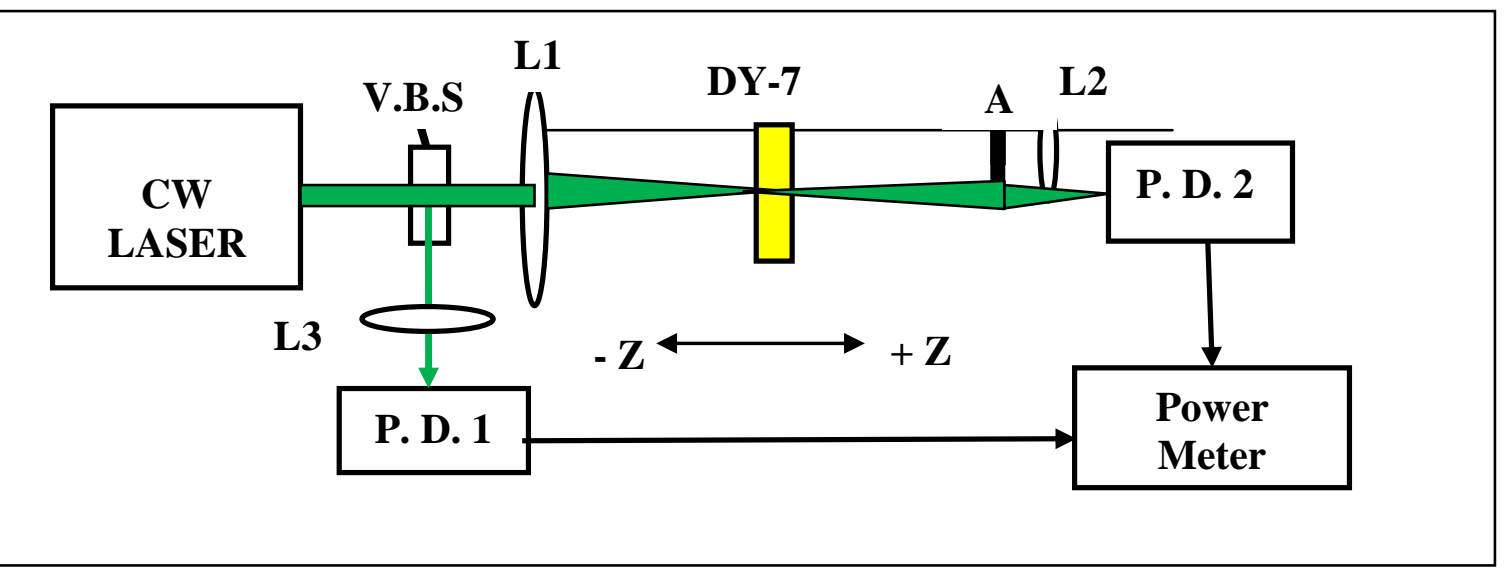

Fig. 7 : Experimental setup for closed aperture Z scan for DY-7 sample.

The experimental setup used for the closed aperture $Z$-scan technique is same as the setup used for open aperture Z-scan except for the output beam from the dye sample is collected through an aperture a fixed hole size instead of collecting entire output beam through collecting lens L2. The diode laser of wavelength $532 \mathrm{~nm}$ is used as the excitation source and the Gaussian beam profile was focused by a convex lens (L1), of focal length, $f=3: 5 \mathrm{~cm}$ to produce a beam waist $\omega_{0}$ of 15 $\mu \mathrm{m}$. The peak intensity of the incident laser beam is $\mathrm{I}_{0}=3: 5 \mathrm{~kW} / \mathrm{cm}^{2}$. The diffraction length, $Z_{\mathrm{R}}$ was found to be $2.5 \mathrm{~mm}$. The schematic of the experimental setup used is shown in figure 7 . The dye sample is translated across the focal region along the axial direction that is the direction of 
the propagation laser beam. The transmission of the beam through an aperture placed in the far field is measured using photo detector fed to the digital power meter. The closed aperture Z-scan plot between $\mathrm{Z}$ in $\mathrm{mm}$ and normalized transmittance for different dye concentration is shown in figure 8.

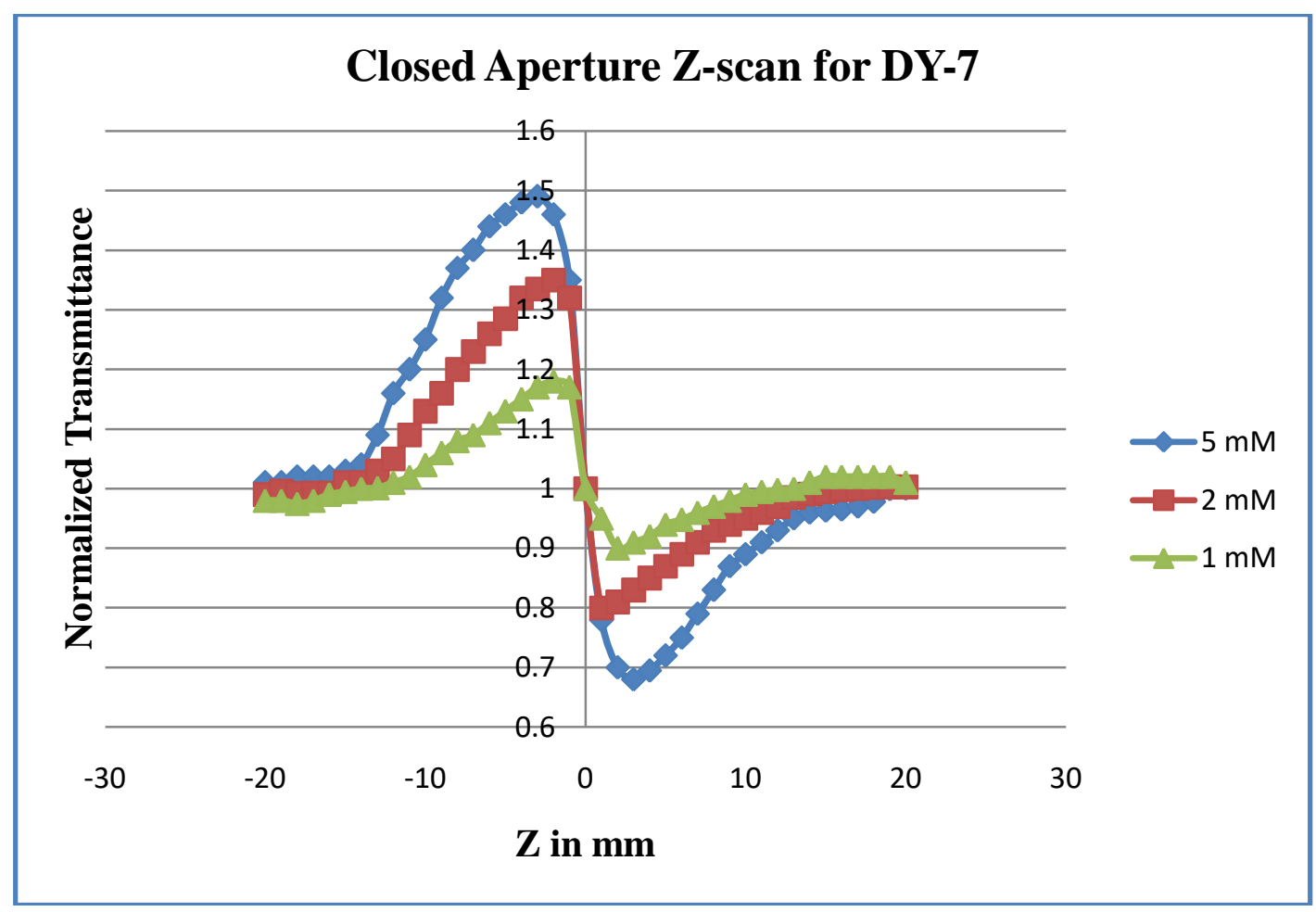

Fig. 8 : Nonlinear refraction of DY-7 using CW $532 \mathrm{~nm} 20 \mathrm{~mW}$ laser beam.

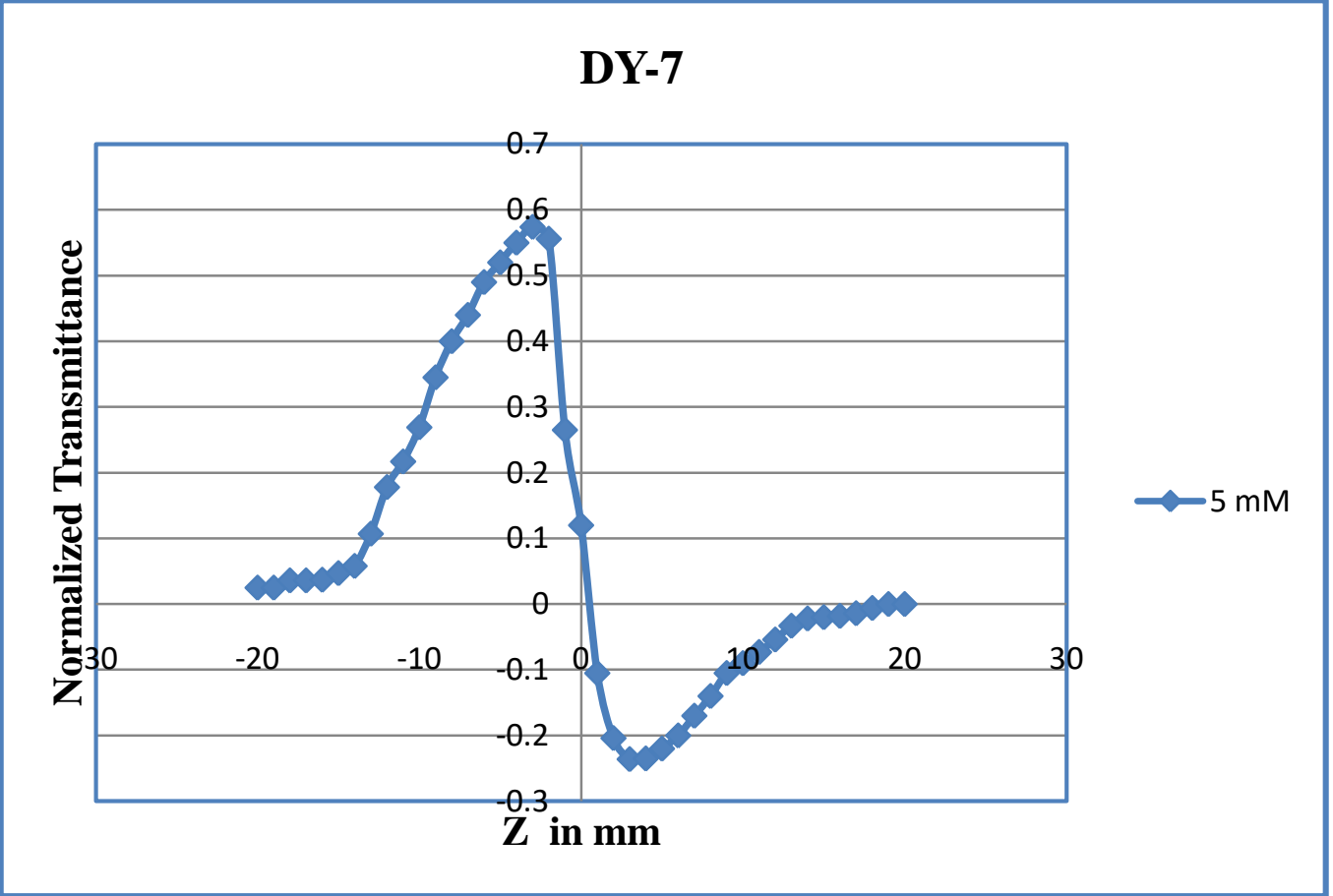

Fig. 9 : Pure nonlinear refraction plot of DY-7 using CW $532 \mathrm{~nm} 20 \mathrm{~mW}$ laser beam. 
The peak followed by a valley-normalized transmittance curve obtained from the closed aperture $Z$-scan data indicates that the sign of the refraction nonlinearity is negative, i.e. self-defocusing. The self-defocusing effect is due to the local variation of the refractive index with temperature. The defocusing effect for the dye in solvent and polymer film shown in figure 8 is attributed to a thermal nonlinearity resulting from the absorption of radiation at $532 \mathrm{~nm}$.

The measurable quantity $\Delta T_{\mathrm{p}-\mathrm{v}}$ can be defined as the difference between the normalized peak and valley transmittances. Since the closed aperture transmittance is affected by the nonlinear refraction and absorption, the determination of $\beta$ is less straightforward from the closed aperture scans. Therefore, it is necessary to separate the effect of nonlinear refraction from that of the nonlinear absorption. A simple and approximate method to obtain purely effective is to divide the closed aperture transmittance by the corresponding open aperture scans. The pure nonlinear refractive index $n_{2}$ is obtained by dividing the closed aperture data by the open aperture data [36]. The pure nonlinear refraction Z-scan curves are shown in figure 9 for the Dy-7 dye in the polymer film. Experimentally determined nonlinear refractive index $n_{2}$ and nonlinear absorption coefficient $\beta$ can be used in finding the absolute value of the third-order nonlinear optical susceptibility. In order to know the contribution from pure PMMA-MA polymer film to the observed nonlinear response, the Z-scan is performed on pure film without DY-7 dye doping. Neither nonlinear absorption nor nonlinear refraction is observed.

\section{Experimental Configuration for Optical Limiting}

The optical limiting effect of the DY-7 dye-doped polymer film is studied by using a $30 \mathrm{~mW}$ CW semiconductor laser at $532 \mathrm{~nm}$. Two experimental setups ate used for the demonstration of optical limiting. In the first experimental setup, the dye sample is placed in the focus of the focusing lens L1 of Z-scan setup. The emergent beam from the dye sample is collected to a photo detector by means of a collecting lens L2 to measure the output power. By fixing the sample position at the focus, the input power is varied and output power is noted. Such experimental setup is named as Optical limiting without an aperture or Type 1 optical limiting. This type of optical limiting study will take care of nonlinear absorption property of the dye sample. In the second experimental setup, an aperture of fixed hole size is used between the dye sample and the collecting lens \& photo detector. The dye sample film is kept at the position where the transmitted intensity shows a valley in closed aperture Z-scan curve [36]. The input laser intensity is varied systematically and the corresponding output intensity values are measured by the photo detector. At very high peak intensities (closer to the focus) we could observe diffraction type pattern with concentric ring structures probably due to self-phase modulation. However, in limiting experiments we have ensured that there is no ring pattern formation by placing the sample away from focus. Such experimental setup is named as Optical limiting with aperture or

Type 2 optical limiting. This type of optical limiting study will take care of nonlinear refraction property of the dye sample.

\section{Case (1) : Optical Limiting without Aperture (Type 1) :}

The pure nonlinear absorption property of the dye sample is measured using this method of optical limiting without aperture at the output side (Type-1 optical limiting). The entire light beam transmitted through the sample is focused by a collecting lens to the photo detector-power meter assembly. The optical limiting effects of the DY-7 dye-doped PMMA-MA films are studied by using a CW laser source. The experimental set-up for the demonstration of type-1 optical limiting is shown in figure 10. The dye sample is kept fixed at the focal point of the lens L1 of aperture Z-scan setup. A variable beam splitter (VBS) is used to vary the input power. By means of a convex lens, the output light beam is made to fall on the photo-detector (PD). The input light intensity is increased systematically and the corresponding output intensity is 
measured by a photo detector. The output power is measured using a power meter. The experiment is performed at different input power and the corresponding output power of transmitted beam is noted and a graph is drawn between input power and output power for different dye concentrations and is shown in figure 11.
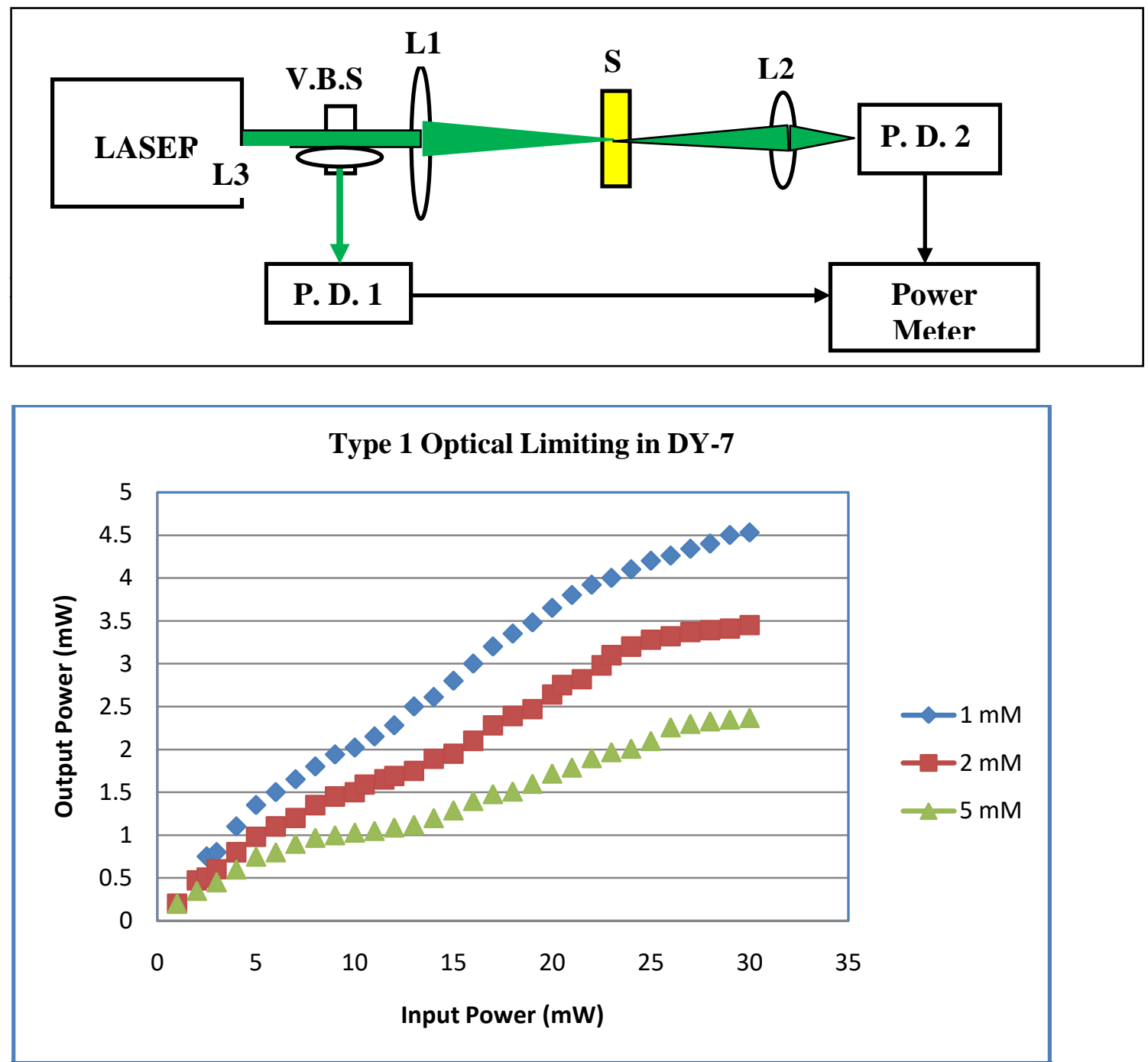

Fig. 11 : Open aperture (Type 1) Optical limiting behavior of DY-7 dye-doped PMMA-MA film at CW $532 \mathrm{~nm}$.

In this case, the intensity of the transmitted output beam is found to vary linearly with the incident input intensity for low values of input but starts to saturate at high incident intensities due to nonlinear two-photon absorption. Hence, after a certain threshold value of the input intensity, the nonlinear absorption of the dye sample becomes dominant, resulting in a limiting of the intensity of output beam. Thus the transmittance recorded by the photo detector remained reasonably constant showing a plateau region.

\section{Case (2) Optical Limiting with Aperture (Type 2) :}

The pure nonlinear refraction property of the dye sample is measured using this method of optical limiting with an aperture at the output side (Type-2 optical limiting). The light beam transmitted through the sample is passed through an aperture A of fixed diameter and then passed through a 
collecting lens L2 to the photo detector-power meter assembly. The optical limiting effects of the DY-7 dye-doped PMMA-MA films are studied by using a CW laser source. The experimental set-up for the demonstration of type-2 optical limiting is shown in figure 12 . The dye sample is kept at the position where the transmitted intensity shows a valley in the closed aperture Z-scan curve. The experiment is performed at different input power and the corresponding output power of transmitted beam is noted and a graph is drawn between input power and output power for different dye concentrations and is shown in figure 13.

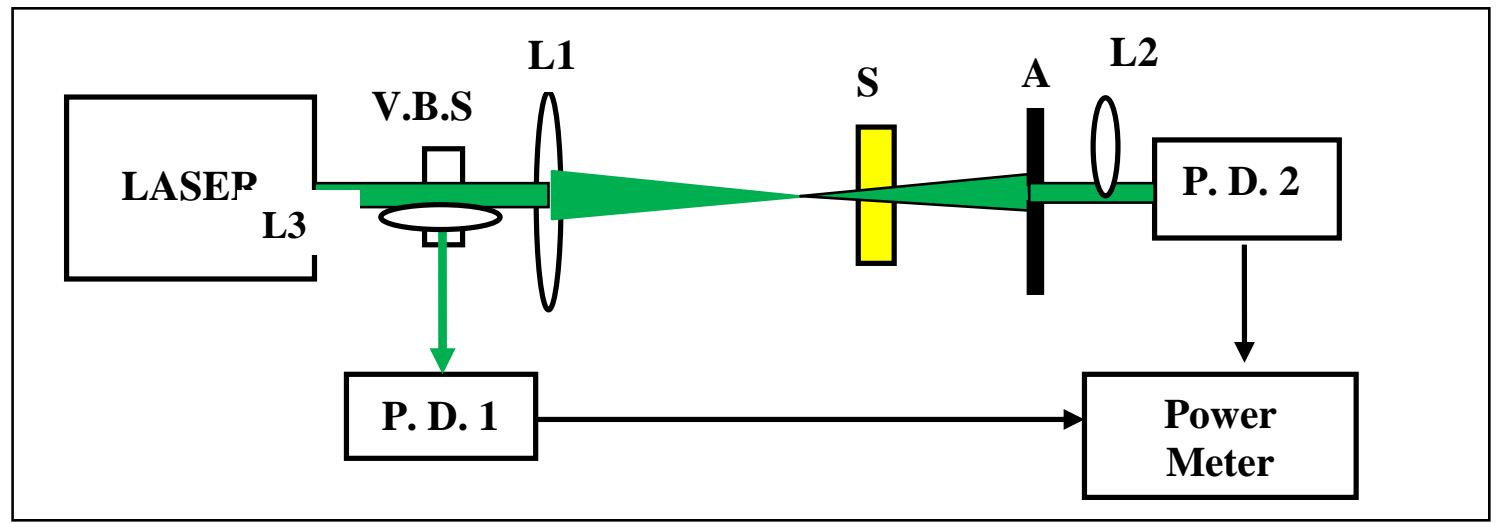

Fig. 12 : Experimental setup for closed aperture Optical limiting for DY-7 doped PMMA-MA using nonlinear refraction.

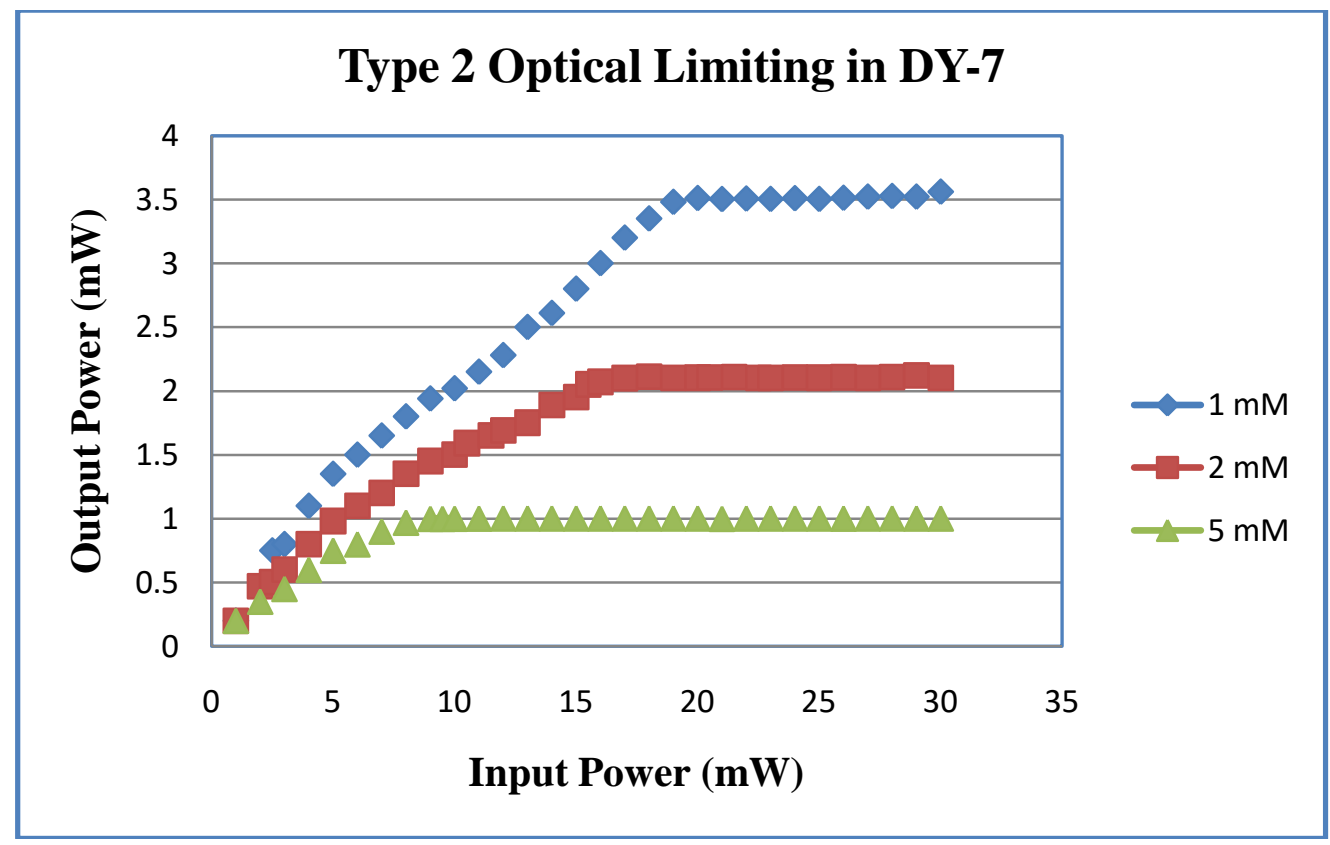

Fig. 13 : Closed aperture (Type 2) Optical limiting behavior of DY-7 dye-doped PMMA-MA film at $\mathrm{CW} 532 \mathrm{~nm}$.

In this case of DY-7 dye sample with defocusing nonlinearity (negative nonlinearity), the intensity of transmitted output beam is found to vary linearly with the incident input intensity for low values of input, but starts to saturate after a certain threshold value due to the fact that the samples starts defocusing the beam, resulting in a greater part of the beam cross-section being cut off by the aperture A placed in between the sample and the collecting lens before the photo- 
detector. Thus the transmittance recorded by the photo detector remained reasonably constant showing a plateau region as shown in figure 13 .

\section{Results \& Discussion}

The optical nonlinearity of DY-7 doped PMMA-MA polymer film is studied using open aperture and closed aperture Z-scan techniques using low power CW laser ar different dye concentrations. The open aperture Z-scan study shows that the sample dye shows a considerable amount of saturable absorption property. But at higher input intensity, the reverse saturation absorption property of the dye in polymer film becomes prominent as shown in Z-scan graph figure 6 . It is seen that the transmission at the focus decreases with increasing sample concentration. At higher concentration, the sample gives better nonlinear optical properties. Absorption saturation in the sample enhances the peak and decreases the valley in the closed aperture $Z$-scan and results in distortions in the symmetry of the $Z$-scan about $Z=0$. The closed aperture Z-scan study shows that the dye sample shows a considerable amount of negative nonlinear refraction and hence defocuses the laser beam passes through it. Table 1 shows the experimentally calculated Nonlinear optical parameters of DY-7 dye-doped PMMA-MA film at $532 \mathrm{~nm}$.

Table 1 : Nonlinear parameters for DY-7 dye-doped PMMA-MA film at $532 \mathrm{~nm}$.

\begin{tabular}{|c|c|c|c|}
\hline S. No. & Parameter & Dye concentration & DY -7 \\
\hline \multirow[t]{3}{*}{1} & \multirow{3}{*}{$\begin{array}{l}\Delta T_{p-v} \\
\text { (The difference between the normalized } \\
\text { peak and valley transmittance) }\end{array}$} & $1 \mathrm{mM}$ & 0.29 \\
\hline & & $2 \mathrm{mM}$ & 0.55 \\
\hline & & $5 \mathrm{mM}$ & 0.82 \\
\hline \multirow[t]{3}{*}{2} & \multirow{3}{*}{$\begin{array}{l}\mathrm{n}_{2} \times 10^{-7}\left(\mathrm{~cm}^{2} / \mathrm{W}\right) \\
\text { (Pure nonlinear refractive index } n_{2} \text { is } \\
\text { obtained by dividing the closed aperture } \\
\text { data by the open aperture data) }\end{array}$} & $1 \mathrm{mM}$ & -0.21 \\
\hline & & $2 \mathrm{mM}$ & -0.52 \\
\hline & & $5 \mathrm{mM}$ & -0.79 \\
\hline \multirow[t]{3}{*}{3} & \multirow{3}{*}{$\begin{array}{l}\beta \times 10^{-3}(\mathrm{~cm} / \mathrm{W}) \\
(\text { Using equation } 3)\end{array}$} & $1 \mathrm{mM}$ & -0.24 \\
\hline & & $2 \mathrm{mM}$ & -0.29 \\
\hline & & $5 \mathrm{mM}$ & -0.36 \\
\hline \multirow[t]{3}{*}{4} & \multirow{3}{*}{$\begin{array}{l}\Delta \mathrm{n}=\mathrm{n}_{2} \mathrm{I}_{0} \\
\left(\times 10^{-4}\right)\end{array}$} & $1 \mathrm{mM}$ & -0.735 \\
\hline & & $2 \mathrm{mM}$ & -1.820 \\
\hline & & $5 \mathrm{mM}$ & -2.765 \\
\hline
\end{tabular}

Optical limiting behavior Disperse Yellow-7 dye-doped PMMA-MA polymer films under low power CW laser excitation for different dye concentrations are studied. The mechanism responsible for optical limiting is mainly attributed to the mechanism of reverse saturation absorption in case of DY-7, which further increased with thermally induced nonlinear refraction. The defocusing effect observed in DY-7 dye samples under CW illumination is utilized to demonstrate their optical limiting action. Based on their nonlinear refractive index, the dyes in PMMA-MA matrix behave as good optical limiters even at low powers. These results are quite encouraging for possible applications in nonlinear optical devices [43-44].

The mechanism responsible for optical limiting is mainly attributed to the mechanism of reverse saturation absorption in case of DY-7, which further increased with thermally induced nonlinear refraction. The defocusing effect observed in DY-7 dye sample under CW laser beam illumination is utilized to demonstrate their optical limiting action. Based on their nonlinear refractive index, the dyes in PMMA-MA matrix behave as good optical limiters even at low powers. These results are quite encouraging for possible applications in nonlinear optical devices. 
Table 2 : Optical limiting Regions in Dye-doped Polymer film at $532 \mathrm{~nm}$ CW laser beam.

\begin{tabular}{|c|c|c|c|c|c|}
\hline $\begin{array}{l}\text { S. } \\
\text { No. }\end{array}$ & $\begin{array}{ll}\text { Sample } & \& \\
\text { Type } & \text { of } \\
\text { Limiting } & \end{array}$ & $\begin{array}{l}\text { DY-7 dye } \\
\text { Concentration }\end{array}$ & $\begin{array}{l}\text { Linear Region } \\
(\mathrm{mW})\end{array}$ & $\begin{array}{l}\text { Active Region } \\
(\mathrm{mW})\end{array}$ & $\begin{array}{l}\text { Saturation } \\
\text { Region }(\mathrm{mW})\end{array}$ \\
\hline \multirow[t]{3}{*}{1} & \multirow{3}{*}{$\begin{array}{l}\text { DY-7 in } \\
\text { PMMA-MA } \\
\text { Type } \\
\text { Limiting } \\
\text { configuration }\end{array}$} & $1 \mathrm{mM}$ & $1-5 \mathrm{~mW}$ & $5-23 \mathrm{~mW}$ & $\begin{array}{ll}23 & \mathrm{~mW} \\
\text { onwards } & \\
\end{array}$ \\
\hline & & $2 \mathrm{mM}$ & $1-8 \mathrm{~mW}$ & $8-25 \mathrm{~mW}$ & $\begin{array}{ll}25 & \mathrm{~mW} \\
\text { onwards } & \\
\end{array}$ \\
\hline & & $5 \mathrm{mM}$ & $1-10 \mathrm{~mW}$ & $10-26 \mathrm{~mW}$ & $\begin{array}{ll}26 & \mathrm{~mW} \\
\text { onwards } & \end{array}$ \\
\hline \multirow[t]{3}{*}{2} & \multirow{3}{*}{$\begin{array}{l}\text { Type } \\
\text { Limiting } \\
\text { configuration }\end{array}$} & $1 \mathrm{mM}$ & $1-7 \mathrm{~mW}$ & $17-18 \mathrm{~mW}$ & $\begin{array}{ll}18 & \mathrm{~mW} \\
\text { onwards } & \\
\end{array}$ \\
\hline & & $2 \mathrm{mM}$ & $1-13 \mathrm{~mW}$ & $13-15 \mathrm{~mW}$ & $\begin{array}{ll}15 & \mathrm{~mW} \\
\text { onwards } & \\
\end{array}$ \\
\hline & & $5 \mathrm{mM}$ & $1-8 \mathrm{~mW}$ & $8-10 \mathrm{~mW}$ & $\begin{array}{ll}10 & \mathrm{~mW} \\
\text { onwards } & \end{array}$ \\
\hline
\end{tabular}

Table 3 : Concentration dependence of saturated output power in DY-7 dye-doped in PMMA-MA film.

\begin{tabular}{|l|l|l|l|}
\hline S. No. & $\begin{array}{l}\text { Dye Concentration } \\
(\mathbf{m M})\end{array}$ & $\begin{array}{l}\text { Type 1 Optical Limiting } \\
\text { Saturated Output Power } \\
(\mathbf{m W})\end{array}$ & $\begin{array}{l}\text { Type 2 Optical Limiting } \\
\text { Saturated Output Power } \\
(\mathbf{m W})\end{array}$ \\
\hline 1 & $1 \mathrm{mM}$ & 4.50 & 3.50 \\
\hline 2 & $2 \mathrm{mM}$ & 3.40 & 2.10 \\
\hline 3 & $5 \mathrm{mM}$ & 2.32 & 1.02 \\
\hline
\end{tabular}

Both type 1 and type 2 optical limiting effects show an increase with increasing the concentration of the dye in the polymer film as shown in figure $11 \&$ figure 13 . The optical limiting responses of the low dye concentration films are generally much weaker than those of high concentrated films, while high concentrated films exhibit strong optical limiting. This indicates that the number density of dye molecules in the laser beam is the main factor affecting the output clamping level. From the threshold intensity for optical limiting, it can be seen that the optical power limiting threshold is inversely proportional to the dye concentration in the film. The limiting experiment shows that as the concentration increases, a reduction in linear transmittance as well as the output clamping level. The experimentally determined optical limiting saturated output power values are shown in Table 3. The results are comparable to some of the reports of low power optical limiting [45-46].

In the case of optical limiter with aperture, as observed in our experiment and in other published results, it is seen that at the valley positions, the limiter works at very low powers as the selfdefocusing effect is enhanced by the thermal effect which is closely related to the absorptive properties of the dyes used. Thus it can be suggested that the best position for a sample, when used for optical limiting based on self-defocusing is at the valley point of the Z-scan curve.

\section{Conclusion}

The nonlinear absorption, nonlinear refraction properties of prepared films of Disperse yellow-7 dyes doped in Polymethyl methacrylate methacrylic acid (PMMA-MA) are studied using the Zscan experimental method. The optical limiting properties of these films are also studied at different input fluency. Good films are grown without any crack using the hot-press technique. 
Linear absorption and nonlinear absorption using z-scan are studied. we have studied the nonlinear optical properties like nonlinear absorption and nonlinear refraction of an azo dye disperse yellow-7 (DY-7) doped in Polymethyl methacrylate-methacrylic acid (PMMA-MA) polymer matrix using open aperture and closed aperture Z-scan experimental method. Finally, the optical limiting properties of these films are also studied at different input power using continuous wave $(\mathrm{CW})$ laser beams of $532 \mathrm{~nm}$ wavelength. It is found that the type of nonlinear absorption depends on the intensity of input beam. Disperse orange has shown saturation absorption at lower input irradiance and then reverse saturation absorption at higher irradiance. On the other hand, Disperse yellow has shown Reverse saturation absorption only at $532 \mathrm{~nm}$.

\section{References}

[1] Perry, J.W., Mansour, K., Mander, S.R., Perry, K. J., Alverez, D. \& Choong, I. (1994). Enhanced reverse saturable absorption and optical limiting in heavy-atom-substituted phthalocyanines, Opt. Lett., 19, 625-627.

[2] Blau, W., Byrne, H., Dennis, W. M. and Kelly, J. M. (1985). Reverse Saturable absorption in tetraphenylporphyrines, Opt. Commun., 56, 25-29, 1985.

[3] Sevian, A., Ravikanth, M. and Kumar, G.R. (1996). Optical limiting in short chain basket handle porphyrins, Chem. Phys. Lett., 263, 241-246, 1996.

[4] Sharma, S., Mohan, D., \& Ghoshal, S. K. (2008). Measurement of nonlinear properties and optical limiting ability of Rhodamine 6G doped silica and polymeric samples. Optics Communications, 281(10), 2923-2929.

[5] Castillo, J., Kozich, V.P. and Marcano, A.O. (1994). Thermal lensing resulting from one and two-photon absorption studied with a two color time-resolved Z-scan. Opt. Lett., 19, 171-173.

[6] Krishnamurthy, R. R., \& Alkondan, R. (2010). Nonlinear characterization of Mercurochrome dye for potential application in optical limiting. Opt. Appl. XL, 187-196.

[7] Rekha, R. K., \& Ramalingam, A. (2009). Nonlinear characteristic and optical limiting effect of oil red O azo dye in liquid and solid media. Journal of Modern Optics, 56(9), 1096-1102.

[8] Muto, S., Kubo, T., Kurokawa, Y. and Suzuki, K. (1998). Third-order nonlinear optical properties of Disperse Red 1 and Au nanometer-size particle-doped alumina films prepared by sol-gel method. Thin Solid Film, 322, 233-237.

[9] Zidan, M. D., \& Ajji, Z. (2011). Optical limiting behavior of disperse red 1 dye doped polymer. Optics \& Laser Technology, 43(5), 934-937.

[10] Brzozowski, L. \& Sargent, E. H. (2001). Azobenzenes for photonic network applications: Third-order nonlinear optical properties. J. Material Science: Materials in Electronics, 12, 483489.

[11] Naseema, K., Manjunatha, K. B., Sujith, K. V., Umesh, G., Kalluraya, B., \& Rao, V. (2012). Third order optical nonlinearity and optical limiting studies of propane hydrazides. Optical Materials, 34(11), 1751-1757.

[12] Venugopal Rao, S., Naga Srinivas, N.K.M. and Narayana Rao, D. (2002). Nonlinear absorption and excited state dynamics in Rhodamine B studied using Z-scan and degenerate four wave mixing techniques, Chem. Phys. Lett., 361, 439-445.

[13] Umakanta Tripathy, R., Justin Rajesh, R., Prem, B.B. \& Subrahamanyam, (2002). Optical nonlinearity of organic dyes as studied by Z-scan and transient grating techniques. Procs. Indian Acad. Sci. (Chem. Sci.), 114(6), 557-564. 
[14] Sukumaran, V. S., \& Ramalingam, A. (2011). Third order optical nonlinearities and spectral characteristics of methylene blue. Journal of Quantum Information Science, 1(02), 69.

[15] Chen, Q., Kuang, L., Sargent, E.H. and Wang, Z.Y. (2003). Ultrafast nonresonant third-order optical nonlinearity of fullerene-containing polyurethane films at telecommunication wavelengths, Appl. Phys. Lett., 83, 2115-2117.

[16] Zidan, M. D., Ajji, Z., Allaf, A. W., \& Allahham, A. (2011). Investigation of the optical limiting properties of acid blue 29 in various solvents. Optics \& Laser Technology, 43(7), 13471350.

[17] Dharmadhikari, A. K., Roy, B., Roy, S., Dharmadhikari, J.A., Mishra, A. and Kumar, R.G. (2004). Higher-order optical nonlinearities in 4-dimethylamino-N-methyl-4-stilbazolium tosylate. Opt. Commun., 235, 195-200.

[18] Ganeev, R. A., Baba, M., Morita, M., Ryasnyansky, A.I., Suzuki, M., Turu, M. and Kuroda, H. (2004). Fifth-order optical nonlinearity of pseudoisocyanine solution at $529 \mathrm{~nm}, J$. Opt. A: Pure Appl. Opt., 6, 282-287.

[19] Del Nero, J., de Araujo, R. E., Gomes, A.S.L. and de Melo, C. P. (2005). Theoretical and experimental investigation of the second hyperpolarizabilities of methyl orange, J. Chem. Phys., 122(104506), 1-6.

[20] Hassan, Q. M. A., (2015). Optical limiting properties of sudan red B in solution and solid film Optical and Quantum Electronics, 47(2), 297-311.

[21] Aithal, P. S., Singh, R. P. \& Rao, D. N. (2003). Optical limiting due to frequency upconverted fluorescence in DASPB dye doped polymer matrix. Proceedings of SPIE, 4797, 229239.

[22] Singh, R. P., Aithal, P. S. \& Rao, D. N. (2003). Optical limiting studies of Disperse Orange and Disperse Yellow in PMMA-MA matrix. Proc. of SPIE, 4797, 52-58.

[23] Shubhrajyotsna Aithal, Aithal, P. S. \& Bhat, G. K. (2013). Study of Optical Limiting and Optical Phase Conjugation in DASPB dye-doped polymer films, GSTF Journal of Physics and Applications (JPA), 1(1), 15-24. DOI: 10.5176/2335-6901.1.1.3.

[24] Shubhrajyotsna Aithal, Aithal, P. S. \& Bhat, G. K. (2012). Study of Degenerate Four-Wave Mixing in Disperse Orange Dye-doped Polymer Film, Advanced Materials Research Journal, 584, 526-530. doi:10.4028/www.scientific.net/AMR.584.526.

[25] Shubhrajyotsna Aithal, Aithal, P. S. \& Bhat, G. K. (2015), Comparative Study on Azo dyedoped Polymer Films for Optical Phase Conjugation. International Journal of Science and Research (IJSR), 4(4), 436-441. DOI: 10.5281/zenodo.61724.

[26] Shubrajyotsna Aithal, Aithal, P. S. \& Bhat, G. K. (2013). Degenerate four-wave mixing in DASPB dye-doped polymer film, published in Part IV Quantum Optics, Chapter 12, Advances in Laser Physics and Technology, Edited by Man Mohan, Anil Kumar Maini, Aranya A. Bhattacherjee and Anil K. Razdan under the imprint of Foundation Books, Cambridge University Press India Pvt Ltd., pp. 179 - 195, ISBN: 978-93-844634-1-0., DOI : 10.5281/zenodo.62048.

[27] Shubrajyotsna Aithal, Aithal, P. S. \& Bhat, G. K. (2012). Phase Conjugation in Two Photon Absorbing Dye films by Degenerate Four-wave Mixing, $3^{\text {rd }}$ International Conference on Photonics 2012, 1-3 October 2012, Penang, Malaysia. Published in IEEEXplore ISBN: 978-14673-1463-3, pp.235-239. 
[28] Shubrajyotsna Aithal, Aithal, P. S. \& Bhat, G. K. (2012). Study of Degenerate Four-Wave Mixing in Disperse Orange Dye-doped Polymer Film, Advanced Materials Research Journal, 584, 526-530.

[29] Shubrajyotsna Aithal, Aithal, P. S. (2012). Study of Phase Conjugated wave in DASPB dyedoped polymer films, Photonics Global Conference 2012, 13-16, December 2012, Nanyang Technical University, Singapore. In Photonics Global Conference (PGC), Singapore, pp.1-5. IEEE. ISBN : 978-1-4673-2513-4, DOI:10.1109/PGC.2012.6458057.

[30] Shubrajyotsna Aithal, Aithal, P. S. \& Bhat, G. K. (2016). Study of Low Power Degenerate Four-Wave Mixing in Disperse Yellow Dye-doped Polymer Film. International Journal of Engineering Research and Modern Education (IJERME), 1(2), 200-209. DOI: http://dx.doi.org/10.5281/ZENODO.198716.

[31] Van Stryland, E. W., Wu, Y. Y., Hagan, D. J., Soileau, M.J. \& Kamjou Mansour (1988). Optical Limiting with Semiconductors. JOSA B5, 1980-1989.

[32] Yee, K. C. Tou, T.Y. \& Ng, S. W. (1998). Hot-press molded PMMA matrix for solid-state dye lasers. Applied Optics, 37, 6381-6385.

[33] Shubhrajyotsna Aithal, Aithal, P. S. \& Bhat, G. K. (2011). Nonlinear Absorption Studies of Disperse Orange Doped Polymer Film, Trends in Optics and Photonics II, Proceedings of International Conference on Trends in Optics and Photonics, December 7-9, 2011, Kolkata, India. Editors : Ajay Ghosh and Debesh Choudhury, ISBN 978-81-908188-1-0, P. 132-137, (2011), DOI : $10.5281 /$ zenodo.62034.

[34] Shubrajyotsna Aithal, Aithal, P. S. \& Bhat, G. K. (2015). A Review on Sustainable Organic Materials for Optical Limiting Technology. International Journal of Management, IT and Engineering (IJMIE), 5(7), 527-544, DOI : 10.5281/zenodo.62032.

[35] Sheik-Bahae, M., Said, A. A. \& Van Strayland, E. W. (1989). High Sensitivity Single beam n2 Measurement. Opt. Lett., 14, 955-957.

[36] Sheik-Bahae, M., Said, A. A., Wei, T., Hagan, D. J. and Van Strayland, E.W. (1990). Sensitivitive measurement of optical nonlinearities using a single beam. IEEE J. Quantum Electron., 26, 760-769.

[37] Rajashekar, B. Sagar Limbu, Kamarusu Aditya, Nageswara Rao, G. \& Siva Sankara Sai , S. (2013). Azo doped polymer thin films for active and passive optical power limiting applications. Photochem. Photobiol. Sci., 12(10), 1780-1786.

[38] Nagaraja, K. K., Pramodini, S., Santhosh Kumar, A. Nagaraja, H. S., Poornesh, P. \& Dhananjaya Kekuda. (2013). Third-order nonlinear optical properties of Mn doped $\mathrm{ZnO}$ thin films under cw laser illumination. Optical Materials. 35(3), 431-439.

[39] Pradeep, C., Mathew, S., Nithyaja, B., Radhakrishnan, P. and Nampoori, V.P.N., (2014). Studies of nonlinear optical properties of PicoGreen dye using Z-scan technique, Applied Physics A, 115, 291-295.

[40] Shubrajyotsna Aithal, Aithal, P. S. \& Bhat, G. K. (2011). Optical Nonlinearity of Dyedoped Polymer Film using Z-scan Technique. Proceedings of Second International Conference on Photonics 2011, Kota Kinabalu, Malaysia, IEEEXplore, ISBN 978-1-61284-265-3, pp 6266. DOI: 10.1109/ICP.2011.6106884.

[41] Shubrajyotsna Aithal, Aithal, P. S. \& Bhat, G. K. (2011). Study of nonlinear absorption in a dye doped polymer film due to frequency up-converted fluorescence. Proceedings of the International Conference on Laser, Material Science and Communication, Ed. Chatterjee, U. \& 
Chakrabarti, P. K. ISBN : 978-93-80813-14-1, pp. 107-109, (2011), DOI : 10.5281/zenodo.62033.

[42] Shubrajyotsna Aithal, Aithal, P. S. \& Bhat, G. K. (2012). Phase Conjugation in Two Photon Absorbing Dye films by Degenerate Four-wave Mixing, $3^{\text {rd }}$ International Conference on Photonics 2012, Malaysia. IEEEXplore ISBN: 978-1-4673-1463-3, pp - 235-239. DOI : 10.1109/ICP.2012.6379868.

[43] Shubrajyotsna Aithal, Aithal, P. S. \& Bhat, G. K. (2015). A Review On Sustainable Organic Materials for Optical Limiting Technology. International Journal of Management, IT and Engineering (IJMIE), 5(7), 527-544. DOI : 10.5281/zenodo.62032.

[44] Shubhrajyotsna Aithal, \& Aithal P. S., (2016). ABCD analysis of Dye doped Polymers for Photonic Applications, IRA-International Journal of Applied Sciences, 4(3), 358-378. DOI : http://dx.doi.org/10.21013/jas.v4.n3.p1.

[45] Kaladevi, S. Vijayan, C. Kothiyal, M.P. (2004). Opt. Mater. 27, 1606.

[46] Balaji, G. Rekha R. K. \& Ramalingam, A. (2011). Nonlinear Characterization of Safranin O Dye for Application in Optical Limiting. ACTA PHYSICA POLONICA A, 119(3), 359-363. 